\title{
Antecedentes sociológicos del estudio de la comunicación interpersonal
}

\author{
María Rosalía Garza GuzMán \\ Universidad Autónoma de Nuevo León \\ rosaliagarza@yahoo.com.mx
}

\begin{abstract}
Resumen:
El estudio de la comunicación interpersonal en México, es poco atendido por académicos e investigadores de la comunicación. Su atención ha estado centrada en los medios masivos de comunicación, ahora desplazados de alguna manera por las actividades comunicativas realizadas en el ciberespacio. Este nuevo entorno a captado la atención sobre las interacciones interpersonales mediadas por computadora. De este vacío generado en torno del nivel interpersonal dentro del campo académico en México, surge el interés de mostrar la pertenencia de ella dentro de esta área de estudio, objetivo que la investigación. Se siguió una metodología con enfoque hermenéutico, bajo la cual se exploró y analizó el estudio de la comunicación interpersonal para lograr, entre otros aspectos, describir el estado que guarda este nivel dentro del campo de la investigación de la comunicación en México.

En la presente publicación se mostrarán los resultados concernientes al análisis teórico y de contenido desarrollado para identificar las raíces genealógicas de la comunicación interpersonal desde la sociología.

Los resultados muestran que uno de los campos científicos que más han aportado conocimientos al respecto es la sociología. Se exponen algunas de las más importantes aportaciones al estudio de la comunicación interpersonal que provienen de sociólogos; Georg Simmel, George Herbert Mead, Erving Goffman y Jürgen Habermas.

Como parte de las conclusiones del panorama completo de la investigación se comprobó la pertinencia de la comunicación interpersonal dentro del campo académico de la comunicación y la urgencia de la atención cabal por parte de los estudiosos e investigadores mexicanos.
\end{abstract}

Palabras clave: Comunicación Interpersonal; Sociología; Antecedentes Genealógicos de la comunicación.

\section{Background sociological study of interpersonal communication}

\begin{abstract}
:
The study of interpersonal communication in Mexico, is underserved by academics and researchers of communication. His attention was focused on the mass media, now displaced in some way by the communication activities carried out in cyberspace. This new environment has captured the attention of the computer-mediated interpersonal interactions.

This vacuum created around interpersonal level within the academic field in Mexico, there is the interest of showing her membership within this area of study, research objective. It followed a hermeneutic methodology, under which explored and analyzed the study of interpersonal communication to achieve, among other things, describe the state that keeps this level in the field of communication research in Mexico.

This publication will show the results concerning the theoretical analysis and content developed to identify the genealogical roots of interpersonal communication from sociology.
\end{abstract}


The results show that one of the scientific fields that has contributed knowledge about sociology. Are some of the most important contributions to the study of interpersonal communication that come from sociologists; Georg Simmel, George Herbert Mead, Erving Goffman and Jürgen Habermas. As part of the conclusions of the full picture of the investigation found the relevance of interpersonal communication within the academic field of communication and comprehensive emergency care by Mexican scholars and researchers.

Key Words: Interpersonal Communication; Sociology; History Genealogy of communication.

\section{Referencia normalizada:}

Garza Guzmán, M. R. (2014): Antecedentes sociológicos del estudio de la comunicación interpersonal. Historia y Comunicación Social. Vol. 19. Núm. Especial Marzo. Págs. 315-326.

Sumario: Introducción. 2. Metodología. 3. Resultados. 3.1. La sociología como fuente genealógica del estudio de la comunicación interpersonal. 3.1.1. Georg Simmel. 3.1.2. George Herbert Mead. 3.1.3. Erving Goffman. 3.1.4. Jürgen Habermas. 4. Conclusiones y recomendaciones

\section{Introducción}

El interés por entender el fenómeno de la comunicación humana ha sido parte de la búsqueda del saber desde el surgimiento de la humanidad. Podemos observar evidencias que desde los primeros filósofos de los que tenemos conocimiento, como Aristóteles, ya se interesaban en esta área.

La ciencia desde entonces ha recorrido un largo camino, podemos ubicar que, entre los siglos XVI y XVII, a partir de Copérnico, Galileo Galilei, Newton, Bacon, Kepler y Descartes, surge el concepto moderno de ciencia. Ciencia que se construyó sobre dos grandes premisas, las aportadas por Newton y por Descartes (Wallerstein, 1999).

Al cambiar la manera de aproximarse al conocimiento, la filosofía fue considerada por los científicos naturales, cada vez más como un "mero sustituto de la teología" en la que se afirma a priori, verdades imposibles de poner a prueba. Por ello, para los primeros años del siglo XIX, la ciencia, como medio para llegar al conocimiento se separa de la filosofía y además, surge una alternativa para la cual el mundo nunca se ha puesto de acuerdo en un nombre único; a veces llamada las artes, a veces las humanidades (Wallerstein, 1999).

Dentro del campo de la ciencia, las ciencias sociales definirían su propio espacio, quedando atrapados entre dos mundos: lo que es "ciencia" y lo que no lo es, entre el conocimiento positivista y el hermenéutico, entre la lucha de la metodología cuantitativa y cualitativa; cuestión que las ciencias de la comunicación heredaría. De todos estos ajustes, ya para 1945, las ciencias quedarían divididas así: las ciencias naturales; las humanidades y la ciencias sociales (Wallerstein, 1999). 
A mediados del siglo XX, las ciencias de la comunicación se creó a partir de nuevos nombres de áreas "interdiciplinarias", derivadas de la dificultad de hallar claros límites entre los campos de estudios que implicaba un estudio multi-disciplinario pero que defendían su derecho de existir en forma separada de otros ámbitos del conocimiento (Wallerstein, 1999).

A continuación mostramos un esquema general del desarrollo de las ciencias hasta la creación siguiendo centralmente el camino hacia las ciencias de la comunicación (Tabla 1).

Tabla 1. Genealogía de los estudios de la comunicación

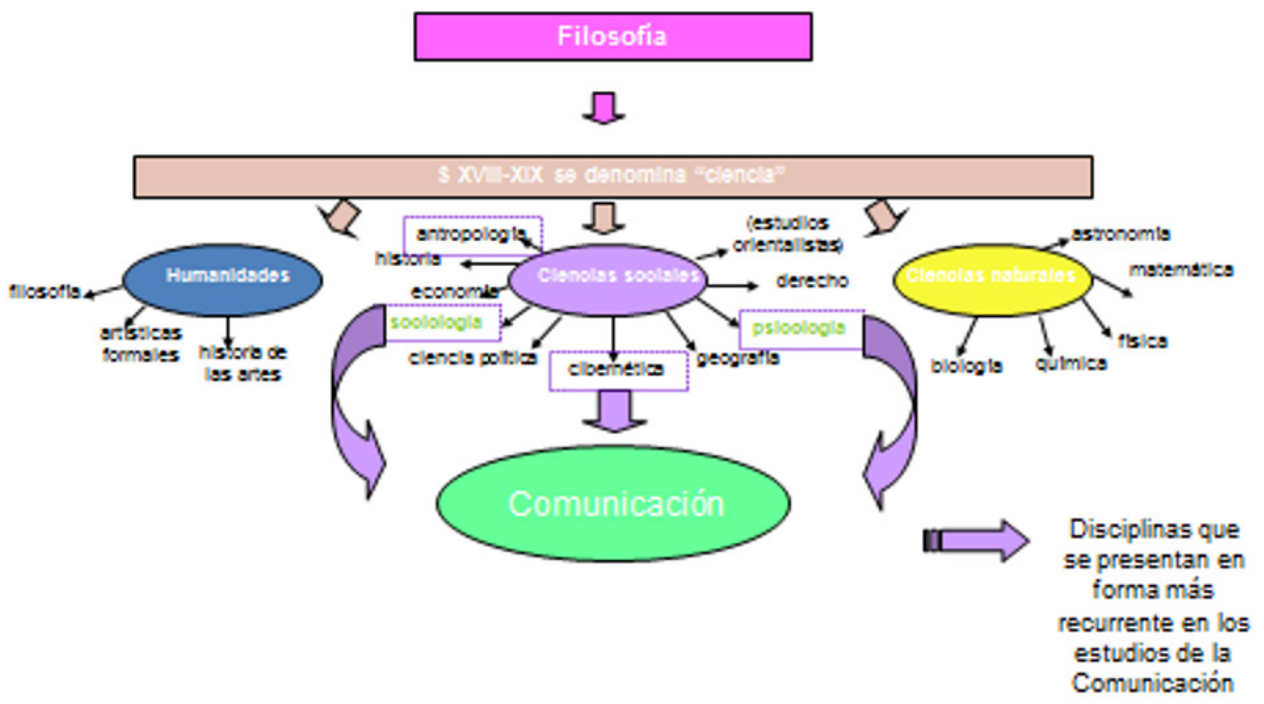

Fuente: Elaboración propia

\section{Metodología}

Bajo una visión hermenéutica, se realizó un proceso indagatorio con el propósito de especificar las propiedades y características del fenómeno analizado: el estudio de la comunicación interpersonal en México.

Este artículo reporta los resultados de uno de los objetivos específicos insertados en una investigación más amplia sobre el estado de la cuestión del estudio de la comunicación interpersonal en México, su pertinencia y sistematización dentro del campo disciplinar de la comunicación (Garza, 2009). 
En dicho objetivo específico se plantea: Elaborar un diagnóstico del estado de la cuestión sobre el pensamiento acerca de la comunicación interpersonal en el ámbito de la reflexión de las ciencias sociales.

Las técnicas utilizadas fueron: a) la investigación bibliográfica con el objetivo de determinar fuentes confiables para el análisis de contenido tanto cualitativo como cuantitativo , b) el análisis cualitativo del discurso, c) el análisis de contenido cuantitativo, para determinar las teorías más relevantes a ser analizadas y d) las entrevistas a expertos.

Para los fines de la investigación, tomaremos el concepto de campo académico manejado por Raúl Fuentes (2005), el cuál parte de la concepción que sobre "campo" científico le ha dado Bourdieu a la palabra champ (campo) del francés, como "espacio" sociocultural de posiciones objetivas donde los agentes luchan por la apropiación del capital común. Fuentes homologa el concepto de campo científico con el campo académico y define que está formado por tres sub- campos: el científico, el educativo y el profesional.

A partir del análisis de bases de datos, libros y revistas especializados en el campo editados en español se e identificaron aquellas fuentes documentales utilizadas para el análisis. En cuanto a las entrevistas con expertos, se ubicaron los estudiosos con mayor grado de publicación sobre el estado de la cuestión del estudio de la comunicación en México, contactando con aquellos con los que se tuvo acceso.

\section{Resultados}

De las tres divisiones de las ciencias (humanidades, naturales y sociales) podemos encontrar antecedentes del pensamiento comunicacional. Mattelart y Mattelart comentan que, ubicados en la confluencia de varias disciplinas, surge el interés sobre los procesos de la comunicación (1997).

En el presente trabajo se reportan los principales hallazgos sobre la genealogía de la comunicación situados en el campo de la sociología, aunque habrá que aclarar que los pensadores mencionados, han dado frutos en diversas corrientes y disciplinas. Siguiendo las aportaciones de estos investigadores, partimos de tomar aquella veta de mayor influencia en el desarrollo de sus pensamientos, así como en la repercusión de los mismos.

3.1. La sociología como fuente genealógica del estudio de la comunicación interpersonal

Las relaciones interpersonales son, para la sociología, los bloques constructores de la estructura social. Desde la sociología interpretativa Max Weber, Alfred Schütz, Peter Ludwing Berger y Thomas Luckmann estudiaron las interacciones intersubjetivas que se establecen en las relaciones humanas. 
En Europa se ubica, a principios del siglo XX, a Georg Simmel, que influyó grandemente en los teóricos de la Escuela de Frankfurt, fundando la Sociedad Alemana de Sociología junto a Max Weber y Ferdinand Tönnies.

En Norteamérica, aunque por la vía de la filosofía y la educación Dewey, filósofo y psicólogo norteamericano, impactó, tanto en el pensamiento de la sociología fenomenológica, como en el desarrollo de la psicología social (Seiler, 2005). Dewey expone que la comunicación permite pasar del plano externo del ser humano, hacia el descubrimiento de sí mismo y hacia su propia revelación, cuyo fruto es la participación social. Sostiene que cuando la comunicación ocurre, los acontecimientos naturales son sometidos a reconsideración y adaptados al discurso, ya sea público o preliminar (pensamiento), convirtiéndo los acontecimientos en objetos con significado (Dewey, 2004).

Este concepto de revelación propia hizo resonancia en diversos teóricos. Se observan en el ámbito sociológico con la Dramatúrgia del sí mismo de Erving Goffman; en el psicológico con la teorías de auto-revelación de Sydney Jourard; y en el modelo utilizado ampliamente en la comunicación organizacional, la ventana de Joharis, desarrollada por Joseph Luft y Harry Ingham.

La corriente crítica del pensamiento representada por la Escuela de Chicago también recibió influencia de las ideas de Dewey. Bajo la visión sociológica fenomenológica de la escuela de Chicago trabajaron en el estudio de la comunicación: Robert Ezra Park, Charles Cooley, Erving Goffman y Herbert Mead (Mattelart y Mattelart, 1997).

También podemos ubicar a Charles Cooley dentro de las corrientes sociológicas antecesoras del campo académico de la comunicación. El concepto de grupo primario, lo que respecta a la socialización del individuo, la dimensión social del yo (looking-glass self), son algunas de sus aportaciones. Entre sus estudios se destaca la importancia de la comunicación en los proceso de socialización y en la articulación de la naturaleza social.Muestra a la comunicación interpersonal como la base de la organización social y en cuyos grupos primarios (familia, barrio, etc.) se conforma la dimensión social del yo.

Su trabajo haría mella en los estudios posteriores dirigidos por Herbert Mead (Interaccionismo simbólico) e Erving Goffman (Dramatúrgia del sí mismo), cuyas obras son altamente referenciadas por los estudiosos de la comunicación en el mundo.

Hiéndanos hacia atrás, las bases de la corriente fenomenológica de la sociología se encuentran en la filosofía de Husserl y en el método de comprensión (verstehen) de Max Weber, cuya corriente de pensamiento sociológica rompe con la estructura de la sociología tradicional al enfatizar en la necesidad de comprender, más que de explicar la realidad. Max Weber establece el concepto de acción social entendiendo por ésta a cualquier actitud o actividad humana -Verhalten- (Weber, 1972; Weber, Durkeim y Parsons, 1984). 
El estudio de la acción social es especialmente importante para la comprensión de la comunicación interpersonal pues deja claro el carácter subjetivo e interactivo de la actividad social, por lo tanto, de la comunicación interpersonal. El concepto de intersubjetividad que sería ampliado por grandes sociólogos como Ludwing Berger, Thomas Luckmann y Alfred Schütz representa uno de los principios de la teoría del interaccionismo simbólico; cimiento de los estudios de la comunicación interpersonal.

Alfred Schütz, en la vía de la intersubjetividad, hace importantes aportaciones al pensamiento social-fenomenológico. Profundiza en el concepto de la significación subjetiva desarrollado por Weber utilizando el concepto de comprensión desarrollado por este último. No obstante, va más allá de una asignación de sentido a las acciones sociales, y describe una "vida cotidiana" en la que se construye el sentido y significación de la realidad. Asimismo, aplica el pensamiento básico de Husserl al análisis de la realidad social. Lo cual resulta cardinal para el estudio de la comunicación interpersonal, pues permite visualizar la vivencia directa del mundo de la realidad social en la que define la situación cara a cara y la relación-nosotros.

Por su lado, Ludwing Berger y Thomas Luckmann, plantean que la realidad se construye socialmente y que, se deben analizar los procesos por los cuales esta construcción se produce. Ellos expresan que la vida diaria se presenta como una realidad interpretada por el ser humano a la que le asigna un significado subjetivo dándole así, coherencia a su mundo (1976).

En sus obras se muestra a la subjetividad como elemento indispensable en el análisis de "la vida cotidiana", dado que ella es como una radiografía del habitual acontecer y es la imagen más visible y reconocible de esa realidad. Las personas tienden a desarrollar patrones repetitivos de comportamiento que se convierten en "hábitos" los cuales les permiten manejar situaciones recurrentes en forma automática, dicen Berger y Luckmann (1976).

Los estudios de la comunicación interpersonal como tal inician con Georg Simmel (Knapp, Daly, Albada y Miller, 2002), a principios del siglo XX, todavía bajo el centro teórico de la sociología. Se expondrán cuatro de las teorías que se consideraron de las más relevantes para el estudio de comunicación interpersonal e identificada como fuentes genealógicas desde la sociología fenomenológica.

\subsubsection{Georg Simmel}

Durante los primeros años de la década de 1900, Georg Simmel trabajó sobre el estudio de las diferentes formas de interacción social, aportando nociones tales como "conocimiento recíproco", "características de la díada", "rituales de interacción", "tipos de relaciones", cuestiones que aún siguen tomándose como fundamentales dentro del estudio de la comunicación interpersonal (Knapp, Daly, Albada y Miller, 2002). 
Para Simmel, resalta la importancia de las interacciones en el desarrollo social del ser humano. Para él, la "sociedad" representa a un número de individuos conectados las interacciones establecidas entre sí. Asimismo, pone énfasis en los significados adquiridos a través del proceso social. Establece que el individuo interioriza los valores, las normas, costumbres, etc., en su conciencia individual, derivado de su proceso de socialización. Y al mismo tiempo, influye en la conformación social al relacionarse con los demás (Simmel, 1971).

Simmel análisis el "secreto" (secrecy) en las relaciones humanas, que surge frecuentemente durante las relaciones interpersonales en la que una persona intencionadamente oculta algo, mientras la otra persona trata de descubrir qué está siendo ocultado (Deflem, 1999; Ritzer y Goodman, 2004); aspecto ampliamente tratado en teorías teorías posteriores como es la dramaturgia del sí mismo de Irving Goffman y las teorías sobre comunicación interpersonal engañosa (Deception Theories) desarrolladas por varios investigadores a mediados de los años novena, entre ellos, David Buller, Judee Burgoon y Gerald Miller.

La "revelación" fue otra de las nociones estudiadas por Simmel. Él observó que la persona elige fragmentos significativos de sus pensamientos para ser compartirlos con su interlocutor y, este conocimiento que tenga uno de otro, es la base sobre la cual se desarrolla la interacción y que afecta la relación. Estos conceptos, en la década de los sesentas seguiría siendo tratado por Sydney Jourard, así como por Joseph Luft y Harry Ingham, los cuales trabajan sobre la auto-revelación.

\subsubsection{George Herbert Mead}

George Herbert Mead concibe el interaccionismo simbólico hacia finales de los años veinte, se observan las interacciones que establecen las personas diariamente y en las cuales compartes significados subjetivos de su propia realidad. Se establece que las estructuras de pensamiento y el concepto de "sí mismo" de las personas (self) surgen de las interacciones con "otros" que le son significativos durante los procesos sociales.

El libro donde se plasma su teoría vería la luz en forma póstuma, Mind, Self and Society publicado en 1939, que impactó enormemente en el desarrollo de esta corriente de pensamiento.

Mead compartió con John Dewey y Charles Horton Cooley su visión desde una filosofía pragmática. Este nuevo enfoque provocaría un giro en los estudios psicológicos de la época, en los cuales se explica la proximidad humana desde el punto de vista comunicativo. Se apartan de la observación del ser humano desde la psicología individual para partir del estudio del comportamiento humano desde un orden social esencial.

George Mead, al tomar el concepto de self, o sí mismo, de Charles Horton Cooley le da atención a la influencia social. Estudia el proceso de cómo el sí mismo es cons- 
tituido a través de la mente, la cognición y el lenguaje, haciendo la distinción en el lado activo del " $P$ " y el pasivo " $m e$ " del self (Mead, 1973).

En esta teoría se parte de la interacción de las personas, considerando que es durante éstas que se crean las estructuras simbólicas sobre las que establecemos acuerdos sobre la concepción de la realidad. Y, es así, como la sociedad es creada. Las interacciones simbólicas permiten a las personas, negociar, manipular, y modificar, hasta cierto punto, la estructura y la realidad social (Mead, 1973).

George Herbert Mead, bajo una teoría evolutiva de la comunicación, hace ver que la comunicación se desarrolla desde un nivel más o menos primitivo, hasta formas más avanzadas de interacción social. A diferencia de los animales, el ser humano cuenta con la lengua, la cual remplaza, pero no suprime, la conversación de gestos. Marca la transición de "no significativo" a la "interacción significativa"; cuestión también tratada posteriormente por Martín Serrano (2007).

Herbert Blumer, discípulo de Mead, trabajó con él y posteriormente continuó en este camino. Blumer es quién acuñó el término de "Interaccionismo Simbólico" y lo utiliza primera vez en un artículo titulado Man and Society publicado en 1937. El término lo utiliza para nombrar un acercamiento desde la perspectiva interaccionista hacia estudio de los grupos y de la conducta humana (Blumer, 1998).

El interaccionismo simbólico se sigue tratando tanto en el campo psicológico como en el sociológico. En el estudio de la comunicación, no solo en el nivel interpersonal, es corriente teórica ampliamente utilizada.

\subsubsection{Erving Goffman}

Erving Goffman desarrolla sus estudios durante la segunda mitad del Siglo XX. Tiene gran influencia de la escuela del Interaccionismo Simbólico. Goffman muestra que las relaciones entre las personas son semejantes a la representación de una obra de teatro en la que las personas, durante sus interacciones tratan, en forma consciente o inconsciente, de manipular la imagen de sí mismo ante los demás.

Así, la comunicación interpersonal es una presentación a través de la cual varios aspectos del "sí mismo" son proyectados al mundo. Las personas suele, a través de sus comunicaciones, mostrar una imagen positiva y ocultar aquellos aspectos de sí mismo que puedan afectar negativamente su imagen ante los otros (Argyle y Trower, 1980; Littlejohn, 1983; Goffman, 2001).

El otro lado de la moneda, es que las personas además de revelarse ante los otros, tratan de obtener información acerca del interlocutor, y pondrán en juego la información que poseen. La información con la que cuentan los interlocutores uno del otro, les permite definir la situación y saber de antemano que es lo que pueden esperar del otro y saber cómo actuar para obtener una respuesta específica (Goffman, 2001).

En la vida diaria las personas muestras una máscara que es parte de la puesta en escena, esto cambiará sutil o radicalmente de acuerdo a la situación o personalidad 
del individuo. Su personalidad será conformada por sus situaciones de vida y las pautas culturales a las que ha estado expuesto. La persona se mostrará ante los demás de acuerdo a la imagen que quiera proyectar, de la manera en que quiera que los demás lo vean. Además, ajustará sus expectativas de rol y estatus que piensa tienen los demás de él o ella.

Goffman tuvo un gran influjo en los análisis sociológicos de la interacción humana, entre ellos, los estudios quinestésico-lingüístico de Ray Birdwhistell y en las teorías de los intercambios sociales iniciadas por Kelley.

\subsubsection{Jürgen Habermas}

Perteneciente a la corriente crítica de la escuela de Frankfurt, Jürgen Habermas inicia sus trabajos en la década de los ochenta. Orienta su metodología de trabajo desde la fenomenología sociológica, desarrollando sus obras bajo una visión hermenéutica.

Habermas apela a la importancia fundamental que para un programa de teoría sociológica es el admitir o rechazar el "sentido" como un concepto fundamental y dice: "Por sentido entiendo paradigmáticamente el significado de una palabra u oración", pues el sentido siempre tiene o encuentra una expresión simbólica, las intenciones del hablante, aunque no existan interacciones, estas intenciones para poder ser expresar claramente siempre podrán adaptar una forma simbólica (1984, Pág.19).

Habermas sostiene que las expresiones pueden ser elementos de un lenguaje natural, o pueden ser un derivado lingüístico como es el lenguaje de los sordomudos o una lengua. La expresión puede ser, tanto lingüística como extraverbal, destacando que en principio y en forma aproximada, todo puede ser reproducido con palabras, pero no todo lo que se puede decir verbalmente, puede ser expresado en forma no verbal (extraverbal).

El término "acción comunicativa" la deriva al distingue entre dos tipos de comportamiento: el regular y el regido por reglas, a esto último lo denomina acción. El comportamiento regular se da con un movimiento de un organismo vivo observable como tal, el cual reproduce su vida adaptándose a su entorno, descubriendo las regularidades a través de una generalización inductiva. La "acción" es aquel comportamiento intencional dirigido $\mathrm{u}$ orientado por normas y reglas que rigen en virtud de un significado intersubjetivamente reconocido.

El comportamiento intencional tiene un contenido semántico, sentido que el ser humano puede distinguir por ser capaz de seguir y entender reglas. La "acción" consiste en darle sentido a las reglas que responden a la intención de un agente que puede orientar su comportamiento a partir de ellas, convirtiendo sus acciones en intencionadas. "El comportamiento y las regularidades comportamentales pueden ser observadas, mientras que las acciones pueden ser entendidas" (Habermas, 1984, Pág. 23). 
Así, establece la diferencia entre acciones con arreglo a fines (instrumentales y/o de elección racional) y las "acciones comunicativas". Las acciones instrumentales buscan el logro de fines y las acciones comunicativas buscan, además, el entendimiento y al ser acciones, se realizan en forma intencionada.

En añadidura, define a la acción comunicativa como una interacción simbólicamente mediada, pues

es orientada por normas sociales obligatorias que definen expectativas reciprocas de comportamiento y que tienen que ser entendidas y reconocidas al menos por dos sujetos agentes (Habermas, 1984).

\section{Conclusiones y recomendaciones}

El identificar las raíces genealógicas sobre los estudios de la comunicación, en general, y sobre la comunicación interpersonal en específico, se vislumbra un campo rico en este aspecto desde las ciencias sociales y en especial desde la sociología. Esto comprueba que no siempre fue la comunicación masiva la única presente en el interés de los científicos de las ciencias sociales y que, su pertenencia al campo académico de la comunicación es pertinente.

Se logró ubicar un sólido cuerpo teórico desarrollado principalmente en Estados Unidos, pero no exclusivo de él, que se define claramente como parte de un campo académico establecido y que responde a las características del estudio de la comunicación, agregándose las peculiaridades del nivel interpersonal, dicho material será motivo de una futura publicación. Estas teorías se han nutrido de los estudios surgidos en otras disciplinas, que en este caso, se ha descrito el campo sociológico. Sin embargo, poseen su propia mirada que se centra en la interacción misma.

El campo científico de la comunicación en México, ha llegado a un grado de especialización que exige transgredir fronteras que vayan más allá de la observación de los fenómenos relacionados con los medios tecnológicos de la comunicación, especialmente en un nivel masivo.

Resulta urgente el incorporar nuevas aristas al campo científico de la comunicación en México. Siendo el estudio de la comunicación interpersonal una de ellas.

Se requiere acercar al comunicólogo al estudio de los fenómenos sociales de la vida cotidiana pues, es a través de las negociaciones de sentido durante las interacciones interpersonales cotidianas que se la realidad social se va construyendo en forma permanente. 


\section{Bibliografía}

ARGYLE, M.; TROWER, Peter (1980). Tú y los demás. México. D.F.: Editoral Harla. BERGER, P. L. y LUCKMANN, T. (1976): La construcción social de la realidad. Buenos Aires. Argentina: Amorrortu.

BLUMER, H. (1998). Symbolic Interactionism. Berkeley. Cal.: University of California Press. BROOM, L. y SELZNICK, P. (1980). Esenciales de sociología. México, D.F.: Compañía Editorial Continental.

CRONK, G. (2005). George Herbert Mead (1863-1931). En FIESER, J.; DOWDEN, B. The Internet Encyclopedia of Philosophy. Disponible en http://www.iep.utm. edu/m/mead.htm. [16-04-2012].

DEFLEM, Mathieu (1999): Classical Sociological Theory. Unpublished notes. Disponible en www.mathieudeflem.net. [15-04-2012].

DEUTSCH, M.; KRAUSS, R. M (1994). Teorías en psicología social. México: Paidós.

DEWEY, J. (1925). Excerpts from: Experience and Nature. http://www.scienzepostmoderne.org/OpereComplete/Dewey.John..Experience\%20and\%20Nature\%20 (1925,\%201929).pdf. [13-04-2012].

FUENTES, R. (2005). El campo académico de la comunicación en México como objeto de análisis auto-reflexivo. En: LOZANO, J. C. (ed.) (2005). La comunicación en México: Diagnósticos, balances y retos. Monterrey, México: Instituto Tecnológico y de Estudios Superiores de Monterrey; Consejo Nacional para la Enseñanza y la Investigación de las Ciencias de la Comunicación, A.C. p. 29-63.

GARZA, M. R. (2009). La comunicación interpersonal dentro del campo académico de la comunicación: Pautas para una sistematización. En: Global Media Journal en español, Otoño 2009. Monterrey, México: Tecnológico de Monterrey. p. 49-60. (2012). Las relaciones interpersonales, entramado para la construcción de la sociedad. En: HINOJOSA, L. (Coord.) (2012). Estudios de cultura, comunicación y tecnologías de la información (Vol. V). España: Comunicación Social. p. 42-57.

GOFFMAN, E. (2001). La presentación de la persona en la vida cotidiana. Buenos Aires, Argentina: Amorrortu Editores.

HABERMAS, J. (1984). Teoría de la acción comunicativa: complementos y estudios previos. Madrid, España: Colección Teorema.

KNAPP, M. L.; DALY; J. A.; ALBADA, Kelly F. y MILLER Gerald R. (2002). Background an Current Trends in the Study of Interpersonal Communication. En: KNAPP, M.; DALY J. A. (edits.) (2002). Handbook of Interpersonal Communication. U.S.A.: Sage Publications. p. 3-20.

LITTLEJOHN, Stephen W. (1978). Theories of Human Communication. U.S.A.: Charles E. Merrit Publishing Company.

(1983). Theories of Human Communication. Belmont, California: Wadsworth Publishing Company.

MARTÍN, J. (2007). Teoría de la comunicación. La comunicación, la vida y la sociedad. España: McGraw Hill.

MATTELART, A.; MATTELART, M. (1997). Historias de la teoría de la comunicación. España: Paidós. Barcelona. 
MEAD, G. (1973). Espiritu, persona y sociedad. Ediciones Paidós Ibérica. Buenos Aires, Argentina.

RITZER, G.; GOODMAN, D. (2004). Sociological Theory. U.S.A.: McGraw-Hill.

SEILER, Robert (s/a): Media studies, critical theory, and cultural studies. Disponible en: http://www.ucalgary.ca/ rseiler/hardt.htm. [24-04-2012]. DEWEY, J. (2004). Democracia y educación. Una introducción a la filosofía dela educación. Secxta edición. Madrid: Ediciones Morata.

SIMMEL, G. (1984). On individual and social forms. Chicago: The University of Chicago.

SCHÜTZ, Alfred (1993). La construcción significativa del mundo social. Introducción a la sociología comprensiva. España: Ediciones Paidós.

WEBER, M. (1986). Ensayos de sociología contemporánea, Selección e introducción de H. Gerth y C. Wright Mills. Barcelona, España: Ediciones Martínez Roca.

WEBER, M.; DURKEIM, E.; PARSONS, T. (1984). Introducción a la sociología. México: Ediciones Quinto Sol.

WALLERSTEIN, E. (1999). La historia de las ciencias sociales. México, D.F.; Universidad Nacional Autónoma de México; Centro de Investigaciones Interdisciplinarias en Ciencias y Humanidades.

\section{La autora}

Obtuvo los grados de Licenciatura en Ciencias de la Comunicación por el Tecnológico de Monterrey, Maestría en Ciencias de la Comunicación por la UANL, Maestría en Desarrollo Humano por la Universidad Iberoamericana y Doctorado en Comunicación Social por la Universidad de La Habana. Profesora de la Facultad de Ciencias de la Comunicación de la U.A.N.L. Miembro del Cuerpo Académico "Estudios de cultura, comunicación y tecnologías de información". Asesora Titular y líder de Proyecto de Investigación en la Escuela de Graduados en Educación del Tecnológico de Monterrey. Miembro de la Cátedra de Investigación de Innovación en Tecnología y Educación del Tecnológico de Monterrey. 